\title{
La investigación en familias de personas con discapacidad
}

\author{
Research in families of people with disabilities \\ Investigação em famílias de pessoas com deficiência
}

Ermilda Almario Ordoñez

Licenciada en pedagogía infantil

Estudiante de la maestría en educación para la inclusión

Universidad Surcolombiana

ermialmario@outlook.com

\section{Resumen}

El presente artículo tiene como objetivo analizar el estado de las investigaciones que se han llevado a cabo recientemente frente a la familia de personas con discapacidad, a partir de una revisión bibliográfica realizada mediante seis categorías, conformadas por: la familia ante la discapacidad, personas con discapacidad, vida familiar, la familia como eje principal para potenciar la autodeterminación en personas con discapacidad, apoyo familiar y profesional y finalmente estrategias y políticas. Se realizó búsquedas en bases de datos como Search, Pubmed, Publindex, Scienceresearch.com, Dialnet, FreeFullPDF y Google Académico, con el referente "familias de personas con discapacidad" o "families of people with disabilities" recopilando 41 artículos, 34 de estos pertenecientes al periodo 2010-2016 y 7 artículos entre el 2000-2009. Se observa una periodicidad estable en la investigación de familias, teniendo como enfoque las diferentes dimensiones y perspectivas que tiene la discapacidad, al igual que la influencia que ejercen la familia y sociedad cercana, develando que la preocupación radica en no encontrar herramientas claras para apoyar, intervenir y empoderar a las familias, y solucionar los diferentes retos que se tejen alrededor de la misma. Las investigaciones de familias de personas con discapacidad brindan nuevas opciones de investigación, a partir de sus resultados, elementos teóricos y estrategias prácticas; para avanzar en el camino de la inclusión social.

Palabras claves: familia, discapacidad, apoyo profesional, estrategias.

\begin{abstract}
This article aims to analyze the status of the investigations that have been carried out recently about the family of people with disabilities, from a literature review from six
\end{abstract}


categories, made up of: the family to disability, people with disabilities, family life, the family as the backbone to promote self-determination for people with disabilities, family and professional support and finally strategies and policies. Searches were performed in databases such as Search, Pubmed, Publindex, Scienceresearch.com, Dialnet, FreeFullPDF and Google Scholar, with the reference "families of persons with disabilities" or "families of people with disabilities" gathering 41 articles, 34 of these belonging to the period between 2010-2016 and 2000-2009 7 articles. A stable frequency is observed in the investigation of families, with the approach the different dimensions and perspectives with the disability, as well as the influence of family and close partnership, revealing that the concern is not finding clear support tools, intervene and empower families, and solve the different challenges that are woven around it. Investigations of families of persons with disabilities provide new research options, from the results, theoretical elements and practical strategies. To advance on the path of social inclusion.

Keywords: family, disability, professional support strategies.

\section{Resumo}

Este artigo visa analisar a situação das investigações que foram realizadas recentemente contra a família das pessoas com deficiência, a partir de uma revisão da literatura por seis categorias, constituídos de: a família à deficiência, pessoas com deficiência, a vida familiar, a família como eixo principal para promover a auto-determinação para pessoas com deficiência, familiares e apoio profissional e, finalmente, estratégias e políticas. pesquisas foram realizadas em bancos de dados, tais como Search, Pubmed, Publindex, Scienceresearch.com, Dialnet, FreeFullPDF e Google Scholar, com a referência "famílias das pessoas com deficiência" ou "famílias das pessoas com deficiência" reunindo 41 artigos, 34 delas pertencente ao período de 2010-2016 e 7 itens entre 2000-2009. uma frequência estável é observada na investigação de famílias, com a aproximação das diferentes dimensões e perspectivas que têm deficiência, como a influência da família e estreita parceria, revelando que a preocupação não é encontrar ferramentas de apoio claras, intervir e capacitar as famílias, e resolver os diferentes desafios que são tecidas em torno dele. Investigações das famílias das pessoas com deficiência proporcionar novas opções de pesquisa, com base em seus resultados, elementos teóricos e estratégias práticas. Para avançar no caminho da inclusão social.

Palavras-chave: estratégias de família, de invalidez, de apoio profissional.

\section{Introducción}

El reconocimiento y la importancia de enfatizar en la familia como unidad fundamental en la construcción de sociedad, ha sido objeto de estudio de innumerables trabajos investigativos. Según Núñez (2008) la familia funciona como una unidad interactiva; en el momento en que uno de sus integrantes afronte condiciones o experiencias adversas a su cotidianidad, perjudicará a los demás miembros de la misma. Partiendo de este hecho, el presente artículo analiza las investigaciones que se han realizado a las familias de personas con discapacidad, a partir de una revisión 
bibliográfica enfocada en las publicaciones entre el 2010-2016, no obstante, por su importancia, se tuvo en cuenta algunos artículos entre el 20002009. Los resultados se analizaron a partir de seis categorías: la familia ante la discapacidad, personas con discapacidad, vida familiar, la familia como eje principal para potenciar la autodeterminación en personas con discapacidad, apoyo familiar y profesional y estrategias y políticas.

\section{La familia ante la discapacidad}

En un mundo perfecto donde la diferencia es estigmatizada, entender la discapacidad como una condición y no como una dificultad o barrera es complejo debido a su trascendencia y evolución a lo largo de la historia, y más cuando está directamente relacionada con el núcleo familiar, como plantea Duran (2011), la influencia e impacto que tiene la discapacidad en la familia, está ligado a las emociones $\mathrm{y}$ funciones que se atribuyen a la crianza, debido a que es la familia el primer entorno que atiende a la persona con discapacidad, lo que hace que las vivencias presenten cambios favorables o desfavorables en el proceso de construcción de significados sobre la discapacidad, que facilita la crianza y cercanía familiar, hecho que solo se logra cuando se ha desarrollado de forma sana las diferentes etapas de duelo. Núñez (2003) plantea que es preciso enfatizar que las familias de personas con discapacidad generalmente experimentan procesos de duelo, que pasan por diferentes fases para aceptar y enfrentar la discapacidad. Por ello, uno de los cambios más comunes a los que se enfrenta la familia es el nacimiento de un nuevo ser, en esta etapa se incrementan las expectativas que tienen hacia el que va a nacer, las creencias e ideologías, al igual que la cultura, la relación familiar y el ritmo de vida que hacen que la homeóstasis familiar se reestructure y se adapte al nuevo ser. La discapacidad supone un impacto en el ámbito familiar, sin importar su procedencia debido a que puede nacer o adquirirla en el transcurso de la vida, el hecho se percibe como algo extraño, inesperado y raro, que fragmenta las expectativas de vida bien sea de la persona o del hijo deseado.

Desde el momento en que se comunica la noticia sobre la discapacidad, la familia afronta diferentes fases que van desde el impacto al recibir la noticia hasta la aceptación. Según Cuadrado, (2010) quien cita a Elisabeth Kübler-Ross, las cinco etapas de duelo que enfrentan las familias con estas condiciones son: negación, ira, negociación, depresión y aceptación. Estas etapas son vivenciadas según la personalidad y vínculo emocional que se tenga con la persona con discapacidad.

La etapa de negación, permite a la familia protegerse del impacto que genera la enfermedad y logra un tiempo para adaptarse a la nueva realidad. Mientras que la ira y el enojo son sentimientos que varían de acuerdo a las características propias de la personalidad. Algunos tendrán control de sus emociones y sobrellevarán mejor la situación, otros por el contrario buscarán un escape para evitar la realidad y otros descargarán su enojo en el familiar con discapacidad o en algún otro miembro de la familia.

Por otro lado, en la etapa de negociación, puede aflorar lo importante y significativo que es la persona con discapacidad para cada integrante de la familia. Permite que la intensidad del enojo disminuya de 
forma progresiva y se inicie a gestar pactos con quien sea el poseedor de su fe, de manera que se logre recuperar el estado de salud de la persona con discapacidad. No obstante, en la etapa de depresión, los dolientes comienzan a comprender la realidad de la enfermedad, experimentan tristeza, miedo e incertidumbre ante lo que implica la discapacidad. Pueden sentir preocupación por cosas que no tienen mayor trascendencia, renegar de sus vidas $y$ mostrarse hostiles y evasivos de la situación. Y finalmente la aceptación, durante esta etapa, los familiares son conscientes de la enfermedad y lentamente la asumen como parte de sus vidas. Por ello, las relaciones intrafamiliares son fundamentales y más cuando el proceso de crianza y fortalecimiento de la persona con discapacidad depende de la misma.

Asimismo Muños (2011) afirma que al igual que los niños con discapacidad presentan necesidades propias, las familias requieren de información, formación y apoyo para tener un mejor proceso de adaptación y logren transitar las etapas de duelo en el ciclo de vida de su familiar con discapacidad, como lo es durante los años escolares, la adolescencia y la adultez. Mercado y García (2010) realizaron 30 entrevistas a profundidad a 24 familiares de personas con discapacidad y 6 entrevistas a profesionales, y afirman que a medida que los hijos crecen, la familia afronta momentos que provocan estrés, generalmente vinculado con ciertas etapas del ciclo vital percibiendo en los padres de familia la ausencia de información sobre la discapacidad e impidiendo un mejor desenvolvimiento de los mismos frente a los diferentes aspectos que rodean este hecho. Del mismo modo
Giulio, Philipov \& Jaschinski (2014) afirman que las familias de personas con discapacidad por lo general tienden a renunciar a sus intenciones de fecundidad, a ser más inestables, a sufrir de dificultades económicas y tienen roles más tradicionales de género, su estado de salud se deteriora $y$ tienen menor bienestar que las familias sin discapacidad.

\section{Personas con discapacidad}

La discapacidad constituye una temática que genera controversias e inquietudes desde diferentes puntos de vista, en lo jurídico, social y personal. Es así como Botero y Londoño (2013) plantean a partir de la interacción con personas que presentan discapacidad física, que la familia y la sociedad en general, brinda un patrón de sobreprotección paternalista $\mathrm{y}$ asistencialista que hace que la persona con discapacidad evada los problemas y tengan dificultad para asumir las consecuencias de sus actos. Asimismo, la valoración subjetiva que éstos realizan sobre su condición de vida en todos los contextos, develan la interacción que existe entre sus recursos, competencias y estado funcional del cuerpo, como una forma de dar respuesta a las condiciones familiares y sociales, determinando así su calidad de vida.

De igual modo, Daly, Louise, Sharek, De Vries, Griffiths, Sheerin, McBennett \& Higgins (2016) agregan que se debe partir de la independencia de cada uno de los miembros de la familia, con el fin de evitar generalizar; potenciando una mejor calidad de vida de las personas con discapacidad.

Pandey \& Agarwal (2013) por medio del análisis documental, develan cómo las 
experiencias, desafíos, barreras y soportes influyen en la persona con discapacidad y en las que requieren de ayuda y apoyo para transitar en su ciclo vital, tal como suele ocurrir en el momento en que el joven con discapacidad transciende a la edad adulta.

Lo anterior se refiere al desarrollo y derecho de la sexualidad, dado que es la familia de la persona con discapacidad quien toma decisiones al respecto; frente a esto Cárdenas (2014) afirma que la sexualidad de personas con discapacidad es un derecho, en el que la familia y profesionales deben brindar apoyo al mismo, teniendo presente la autonomía personal.

Carroscasa (2014) destaca que la discapacidad es un tema que se vivencia con mayor proporción en las personas mayores, en el caso de Europa donde se efectuó una encuesta a 33.281 personas de 50 años en adelante, pertenecientes a 13 países, se evidenció que el $44,1 \%$ de la población afirma tener dificultades en el momento de realizar actividades habituales, debido al deterioro en la salud.

Lo que se busca actualmente para las personas con discapacidad leve $y$ moderada o que tengan habilidades funcionales $\mathrm{y}$ competentes, es lo que plantea Madrigal (2015), tener presente el modelo social como una forma de visualizar a la persona como un sujeto de derechos, capaz y con autoridad de manejar y decidir en su vida, con autonomía e independencia, lo cual conlleva a asumir responsablemente el control de su vida.

\section{Vida familiar}

Todo cambio en la familia y por tanto requiere de reestructuración. Según Silva (2015) "las experiencias familiares sobre la discapacidad mental, no solo se enfocan en el diagnóstico, tratamiento y rehabilitación, sino que dentro de este proceso se ven enmarcados los elementos emocionales, sociales y económicos, generando así un impacto familiar". En el caso de las familias de personas con discapacidad, deben asumir y enfrentar una serie de situaciones e interacciones desconocidas como la discapacidad, el cuidado, la atención médica y terapéutica, el apoyo emocional y económico entre otros, que hacen que la familia desarrolle y fortalezca su capacidad de apertura, flexibilidad, resolutividad y tolerancia a la frustración, de modo que les permita favorecer los cambios y adaptarse a las nuevas dinámicas. Como lo evidencia Sandhu, Ibrahim \& Chinn (2016) al afirmar la búsqueda continua que tiene las madres de personas con discapacidad, basada en el buen vivir.

No obstante, se observan algunas familias donde según Ortega, Salguero y Garrido (2007) los padres se identifican como un grupo diferente de aquellos padres de niños normales, definiendo la discapacidad como un problema con implicaciones sociales, mientras que otros no la catalogan como tal, aspecto que evidencia cambios en la vida de la paternidad, en los juicios de valor y los conceptos de normalidad y anormalidad. Al respecto Madrigal (2015) afirma que los núcleos familiares que cuentan con un miembro en condición de discapacidad tienen prácticas donde predominan mitos, estigmas negativos y desfavorables en la forma en que le visualizan. 
Rea, Acle y Rueda (2014) consideran fundamental trabajar con la familia de personas con discapacidad para que éstas obtengan mayores conocimientos $y$ espacios donde expresen emociones positivas o negativas, brindándole atención adecuada para poder favorecer la adaptación del menor y de la familia ante la discapacidad. Como dice Duran (2011) y Madrigal (2015) la familia es el primer entorno que atiende a la persona con discapacidad, convirtiéndose en el pilar indiscutiblemente más relevante para ser considerado en los diferentes ámbitos de formación, capacitación e información en pro de la persona adulta con discapacidad. Es así como en este proceso, cada uno de los seres y estructura que posee la familia influirá en la vivencia y percepción que tendrán sobre la discapacidad y no obstante en la forma como se afrontará ya que las expectativas de la familia pueden estar centradas en el éxito social o en el afecto y relaciones humanas, dependiendo de la prioridad que esta tenga como una forma de garantizar la homeóstasis familiar. Al respecto Ortega, Salguero, Garrido (2007); Guevara y González (2012); Gonzales, Rueda, Cagigal y Blas (2013) concuerdan al afirmar que la vida familiar transcurre con mayor facilidad cuando se asume la discapacidad, se la acepta y se decide distribuir equitativamente las tareas que la vida conlleva evitando que sea la madre la que asuma todo el trabajo y la responsabilidad, de manera que pueda dedicar también tiempo a sus otros hijos, a quienes debe enseñar a comprender las necesidades que tiene la persona con discapacidad, hacerles partícipes desde pequeños de su atención y cuidado, facilitándoles orientación, formación y apoyo.

De esta manera, según Duran (2011) la convivencia que tiene la familia de la persona con discapacidad, permite la producción de cambios favorables en el proceso de construcción de significados sobre la discapacidad, facilitando la crianza y cercanía. Verdugo y Cruz (2008) sostienen que es importante visualizar los diversos elementos que se deben modificar con el fin de seguir mejorando su calidad de vida.

Según Giulio, Philipov \& Jaschinski (2014) la calidad de vida se centra en las relaciones familiares, estabilidad económica, apoyo profesional y social. De igual forma Carroscasa (2014) plantea que la socialización y unión familiar proporcionan un mejor estado de salud. En este mismo sentido Córdoba, Portilla y Arteaga (2010) afirman que la dinámica familiar interna de las personas con discapacidad leve, está relacionada con la higiene mental de la misma.

\section{La familia como eje principal para potenciar la autodeterminación en personas con discapacidad}

La familia es el escenario donde los seres humanos experimentan y desarrollan sus primeras experiencias de interacción social (León, 2015); allí se dan las bases para crear identidad y se facilita el desarrollo de capacidades y potencialidades individuales que permiten la construcción de un sujeto autónomo. Como lo afirma Mercado y García (2010), la familia es el primer contexto socializador donde evolucionan sus miembros y se desarrollan a nivel afectivo, físico, intelectual y social, por lo que esta debe ofrecer oportunidades que permitan desarrollar habilidades y competencias personales. Partiendo de las particularidades del individuo, según Muños (2011) y Daly et al (2016) las familias deben tener presente que aunque 
su hijo es discapacitado, puede desarrollar diversas habilidades, si se fomentan los puntos fuertes y se apoyan los débiles. Carroscasa (2014) plantea la formación de una red familiar con el fin de brindar bienestar y calidad de vida óptima a la persona con discapacidad como una forma de fortalecer las relaciones familiares.

Manjarres (2012) por medio del análisis que realizó a 60 familias en cuatro departamentos de Colombia, logra concluir que "La familia, debe propiciar y gestionar oportunidades que garanticen el desarrollo máximo de sus potencialidades, el ejercicio de sus derechos, la promoción de proyectos de vida y la inclusión social de la persona con discapacidad". De acuerdo con Bosley \& Kozub (2011) las personas con discapacidad necesitan aprender a interactuar en diferentes contextos fuera de su área de confort, para fortalecer los comportamientos, actitudes y roles que tienen en la sociedad.

En cuanto a Manjarres, León, Martínez, Gaitán (2013) plantean que es necesario que la familia realice búsquedas permanentes de estrategias particulares, soluciones a los retos y desafíos a los que se enfrenta en el proceso de crianza de personas con discapacidad, donde la familia se apropie e impulse a la autonomía. Al respecto Peralta y Arellano (2016) consideran imprescindible:

Que los padres tengan percepciones y expectativas realistas sobre sus hijos con discapacidad y que aprendan estrategias específicas de apoyo dirigidas a potenciar su autodeterminación. Cuando los padres reflexionan sobre sus creencias acerca de la discapacidad, sobre sus expectativas relativas al porvenir de sus hijos, o sobre sus miedos y esperanzas, están más preparados para acompañar a sus hijos con discapacidad sin sustituirlos en el reto que supone vivir la vida por uno mismo.

Es por esto que la familia permite modificar modelos, estereotipos y valores excluyentes que se han naturalizado en la sociedad, transformando la estructura social, en una sociedad inclusiva. En ese caso Lamigueiro (2014) indica que la formación en autodeterminación solo se consigue cuando se logra empoderar a la persona con discapacidad y su familia.

\section{Apoyo familiar y profesional}

No obstante, las actitudes sociales influyen en las vivencias familiares sobre la discapacidad, por lo que es necesario realizar redes de apoyo social y profesional con el fin de que los padres expresen los temores que los invaden con frecuencia, a la vez que se reafirmen los lazos familiares, comprendiendo que la discapacidad no es un tema solo de especialistas, sino de amor y entrega continua, puesto que en algunos momentos la atención puede terminar centrándose únicamente en las necesidades individuales de la persona con discapacidad, y en los efectos negativos que ésta genera, hecho que deja de lado el valor de las fortalezas y las experiencias positivas de toda la familia (Romero y Peralta, 2012). Según Mercado y García (2010) la información constituye un primer paso para la aceptación, integración y superación de los problemas diarios que presenta una persona con discapacidad. Debido a que tanto ella como las familias necesitan de apoyo constante dirigido a las actividades básicas de la vida cotidiana. En este caso Daly et at (2016) reconocen la 
interdependencia de los miembros de la familia $y$ sus implicaciones para su apoyo. Según Peralta y Arellano (2010) "La falta de competencias de las familias para responder a algunas necesidades de sus hijos requiere la puesta en marcha de planes de intervención centrados en la familia que contribuyan a aumentar la autodeterminación de todos sus miembros".

De la misma forma, Marrugat (2005) enfatiza, "ya no se trata de adaptarse a los servicios existentes, sino adaptar los servicios a las necesidades cambiantes de los sujetos". La familia necesita apoyo para el cuidado y educación de la persona con discapacidad, no solo soporte psicológico, sino también práctico además de información y competencias. Según Seliner, Latal y Spirig (2016) por lo general, los padres trabajan fuertemente con el fin de buscar el bienestar de su hijo hospitalizado. Siendo en algunos momentos impotentes al no tener las herramientas idóneas para el trato de su hijo. Hecho que podría mejorar con la colaboración de los especialistas. Como una forma de hacer que el hospital trabaje mancomunado con las familias y logre un mejor servicio en pro del bienestar del niño. Por otro lado, Rodríguez et at (2008) recalca la importancia de apoyar a las familias desde las dimensiones de interacción familiar, salud, seguridad, y rol de padres, los cuales influyen sobre la calidad de vida de la persona con discapacidad. Según Verdugo (2000) el profesional debe proporcionar apoyo e información a aquellos miembros de la familia que quieren colaborar. Debido a que la participación activa de las familias es indispensable para lograr una calidad de vida mejor, en sus miembros y también para ser más eficaces en los apoyos y en los servicios prestados.

Asimismo "para mejorar la colaboración entre padres y profesionales es oportuno analizar las distintas funciones o tareas que los padres han asumido en distintos momentos, y que los profesionales y las organizaciones han esperado que desempeñen" (Verdugo, 2000), igualmente Neely \& Día (2004) señala que para tener buen funcionamiento familiar es importante que los profesionales apoyen las necesidades de toda la familia y no se centren solo en las del niño con discapacidad. Al respecto Peralta y Arellano (2016) afirman que:

Las familias que se sientan capacitadas y que confíen en su capacidad para influir en los servicios y en el desarrollo de sus hijos, son menos vulnerables a los resultados negativos $\mathrm{y}$ en cambio tienen un efecto positivo en la percepción de la calidad de vida familiar.

En concordancia con lo anterior, Guevara y González (2012) plantean:

Es importante que los profesionales adopten un paradigma centrado en la familia para que los servicios de apoyo respondan a las necesidades de todos los integrantes de la familia, de forma individualizada y global. Aunque estas familias pueden compartir experiencias similares, no todas afrontan este acontecimiento de igual manera.

Según Sandhu et al. (2016) las narraciones de las familias pueden proporcionar a los profesionales información significativa acerca de las experiencias de la familia, para visualizar los traumas, esperanzas y expectativas en relación con la prestación del servicio. A 
lo que Fernández, Arias, Gómez y Jarrn (2012) señalan calidad de vida familiar, convirtiéndose en un concepto decisivo para mejorar las capacidades de la familia y a la vez evaluar los resultados de los servicios y apoyos que reciben. Asimismo Arellano y Peralta (2015) señalan que:

Si bien los profesionales, a juicio de los padres, tienen un alto grado de implicación, existe una necesidad real de mejorar sus competencias para interactuar con las familias como un todo, buscar la reciprocidad entre las partes, promover la toma de decisiones o la resolución de conflictos, entre otras. Y, en este proceso, los profesionales necesitan oír de las familias cómo determinadas acciones impactan en su vida diaria. Asimismo, los padres necesitan comprender la perspectiva de los profesionales sobre por qué toman determinadas decisiones. Solo así podrán trabajar conjuntamente hacia una meta global que es mejorar la vida de la persona con discapacidad y la de todos aquellos de su entorno.

\section{Estrategias y políticas}

Es esencial en el proceso de inclusión de parte de la familia y la sociedad conocer los factores relacionales que se tejen alrededor de la persona con discapacidad como lo es el género, el tipo de discapacidad, el proceso de intervención, la edad y proceso evolutivo de la misma. Cada uno de estos aspectos es fundamental para lograr el desarrollo integral de la misma y establecer estrategias, prácticas y normas que contribuyan a la inclusión social de la persona con discapacidad. Según la World Health Organization (2010) en la institucionalización se evidenció deterioro en los niños debido al aislamiento al que eran sometidos. Por el contrario, la gran mayoría de los niños con discapacidad que fueron tratados desde la familia junto con el grupo de profesionales demostraron mejoras en su salud, en su conducta, en el desarrollo, la independencia y habilidades. Madrigal (2015) señala que las instituciones educativas y estatales, las familias, los docentes, los gobernantes y la sociedad deben promover los procesos de inclusión laboral para las personas con discapacidad partiendo de su autonomía. De esa manera, según Gine, Balcells, Roura, Hernández, Mas y Salines (2011):

La necesidad de desarrollar estrategias y protocolos para comunicar a las familias cuanto antes y de forma adecuada (actitud, trato) el diagnóstico y el posible pronóstico, así como mantenerlas informadas del proceso de desarrollo. Atención prioritaria y urgente al hecho de que las familias puedan disponer de servicios de respiro y de "canguros" especializados tanto para hacer frente a las necesidades del día a día como para favorecer su equilibrio emocional ("desconectar"). Promover iniciativas de formación de los padres, sobre todo las relacionadas con hacerles más capaces ("empowerment") de asumir la educación de sus hijos, en particular durante los primeros años.

Asimismo, Manjarres et al (2013) señala que "las familias se encuentran frecuentemente con la carencia de recursos económicos y poca accesibilidad a programas de atención médica y educativa, su voz, reclama acciones por parte del estado que permitan suplir sus necesidades". Hechos que confirma Cárdenas (2014) al develar las dificultades que tienen los profesionales, al momento de interactuar con las personas que presentan alguna 
discapacidad como lo evidenció en su investigación con docentes. Por el contrario Brown, Hoyle \& Karatzias (2016) plantean la necesidad de transcender en las políticas estratégicas centradas en la población con discapacidad, que requieren de mayor compromiso social.

De esa manera, según Mercado y García (2010) la sensibilización juega un papel fundamental no sólo para la incorporación a un puesto de trabajo, sino para la participación e integración de las personas con discapacidad en la sociedad. Por tanto, se deben ofrecer los mecanismos oportunos para que se sientan ciudadanos y ciudadanas de pleno derecho. A la vez Pava (2015) afirma que por medio de las experiencias narradas por la familia, se logra implementar diferentes herramientas y metodologías para superar los temores y revelar los dilemas que se generan en el proceso de inclusión. Al respecto Sáenz (2003) plantea la necesidad de empoderar a la persona con discapacidad junto con la familia para que sean estas las que tengan el protagonismo en las estrategias, organismos, y sociedad que trabajen en pro de su beneficio, y no se descarrile la intervención y apoyo que se destine para los mismos.

Mas y Gine (2012) plantean que la vida familiar se constituye a partir de dos elementos ecológicos como lo son las creencias y los valores, que están inmersos en la sociedad, como una forma de definir políticas y diseñar programas que se ajusten a la realidad de las familias. En cuanto a Lagay, Sanders \& Brophy (2015) indican que la economía está arraigada con la prestación de servicios para personas con discapacidad, lo que hace necesaria la intervención gubernamental.

Según, García, Candido, Saura, Gonzálvez, Vicent y Gómez (2013) y Giulio et al (2014) muchas de las consecuencias de tener un hijo con discapacidad no son inevitables y pueden ser contrarrestadas con políticas adecuadas. Al respecto Araya (2007) plantea:

Las familias son el punto de arranque en la búsqueda de soluciones pues es necesario construir, crear, desarrollar y fortalecer procesos de comunicación que favorezcan la expresión de sentimientos y la apertura para el cambio de actitudes. Ejecutando diseños y políticas igualitarias y universalistas que adviertan que la universalidad se construye desde la diversidad y que, por ende, se requiere una acción estatal que promueva el efectivo cumplimiento de los derechos humanos de las personas con discapacidad y sus familias por medio de la atención a sus particularidades pero libre de los simbolismos asociados a la discapacidad.

Con el fin de llevar a cabo este proceso, Montes y Hernández (2011) consideran necesario crear políticas y servicios centrados en las familias de las personas con discapacidad que les permitan elegir lo más adecuado para ellas y sus hijos de acuerdo a sus prioridades. La formación y capacitación familiar es importante para que la familia sea una verdadera unidad de apoyo.

\section{Resultados}

Las investigaciones analizadas tienen como base la familia de personas con 
discapacidad, se evidencia en las publicaciones del 2000-2009 que se centran en la persona con discapacidad, mientras que las recientes 2010-2016 implementan el enfoque centrado en la familia o calidad de vida como una forma de empoderar y enfrentar esta condición. Lo que devela que en el transcurso de 16 años no ha existido gran avance debido a que casi todas han iniciado desde el mismo punto, sin tener presente los avances de las investigaciones anteriores.

En cuanto a la discapacidad se evidencia un creciente interés por investigar la problemática de personas con discapacidad cognitiva, mientras que la discapacidad visual no fue directamente abordada, comprobando que las investigaciones que giran en torno a la familia de personas con discapacidad, tiende a relacionarse con las discapacidades que requieren de un mayor grado de asistencia, acompañamiento y apoyo.

Todas las publicaciones que son objeto de este análisis, concuerdan al afirmar que el apoyo familiar y profesional, las estrategias y políticas que se establezcan en pro de la población con discapacidad, así como para sus familias son de vital importancia. Debido a la influencia que tienen en el afrontamiento, siendo necesaria la creación de redes de apoyo en la que las familias puedan expresar sus temores e inquietudes, así como encontrar soluciones al respecto.

Las familias requieren de formación, información, apoyo y herramientas con el fin de empoderarlas para que sean ellas las que fortalezcan la autoestima de la persona con discapacidad, permitiendo que tenga autodeterminación.

\section{Conclusión}

La revisión bibliográfica acerca de la familia de personas con discapacidad, permite constatar que los cambios sociales vienen de la mano con la familia. Lo que ha ocasionado el creciente interés por investigar y conocer, las diferentes experiencias y vivencias que giran en torno a la misma, partiendo de los cambios y roles que debe asumir frente a la presencia de la discapacidad en alguno de sus integrantes, siendo oportuno conocer e indagar cada variable que se teje alrededor de estas experiencias para generar estrategias de intervención.

Por otro lado y tras el análisis de las publicaciones basadas en familias de personas con discapacidad, puedo decir que las investigaciones presentan heterogeneidad, respondiendo a necesidades puntuales que surgen en contextos concretos, existiendo una línea de investigación compartida entre los autores, la cual gira entono a la familia como eje garante de los derechos de la persona con discapacidad. No obstante las investigaciones a pesar de compartir el mismo fin, presentan algunas discrepancias en cuanto al método y procedimiento, obteniendo variabilidad en los resultados, quienes al final permiten evidenciar la misma problemática.

En general, los artículos hacen referencia al déficits y limitaciones que tiene la familia en el momento de velar por la calidad de vida de la persona con discapacidad, debido a la falta de información, formación y apoyo para asumirla de forma idónea; acusan la negligencia profesional, la falta de interés de algunas familias, el empoderamiento de los derechos de la persona con 
discapacidad y el desconocimiento de las necesidades familiares.

No obstante se evidencia detención en el proceso, debido a que desde el 2010 hasta el 2016 se tiene un constante énfasis en la familia como eje de la persona con discapacidad, deslumbrando los problemas y posibles soluciones para su intervención, sin evidenciarse la implementación de los mismos fuera de la población objeto de estudio, deteriorando la continuidad de los resultados.

Es así como los esfuerzos realizados para intervenir a la familia que vivencian la discapacidad, requiere de nuevas investigaciones para implementar y desarrollar estrategias que brinden solución respecto al cuidado, manejo y demás variables que surgen en la homeostasis familiar debido al constante cambio que esta tiene, siendo esto crucial para la generación de información al respecto, volviéndose indiscutible señalar lo relevante que es realizar análisis bibliográfico para conocer el estado, avance y problema del tema a investigar, como una forma de aportar nuevos conocimientos.

De ahí, se puede afirmar como conclusión general, que las investigaciones de familias de personas con discapacidad brindan nuevas opciones de investigación, a partir de sus resultados, elementos teóricos y estrategias prácticas para avanzar en el camino de la inclusión social.

\section{Referencias bibliográficas}

Duran, M.; (2011). Familia y discapacidad: vivencias de madres y padres que tiene un/a hijo/a ciego/a o sordo/a. Universidad Central de Venezuela. Venezuela, Caracas. 322p.

Núñez. 2003. La familia con un hijo discapacitado: sus conflictos vinculares, hacia un lineamiento en prevención en salud mental familiar. Arch.argent.pediatr; 101(2). 133 - 142 p.

Cuadrado. 2010. Cinco etapas de cambio. (En línea) consultado en: 09-03-2016. Disponible en: http://factorhuma.org/attachments_sec ure/article/420/c303_cincoetapas.pdf

Muños, A.; (2011). "Impacto de la discapacidad en las familias". Dep. Legal. (38). 1-8.

Mercado, E.; García, L. (2010). "Necesidades sociales de las personas con discapacidad en edad escolar y sus familias". Cuadernos de Trabajo Social, 23, 9-24.

P.; Philipov, D. Jaschinski, I. (2014). "families with disabled children in different European countries las familias con niños discapacitados en diferentes países europeos". Families And Societies. 47 p.

Botero, P.; Londoño, C. (2013). "Factores psicosociales predictores de la calidad de vida en personas en situación de discapacidad física". Acta Colombiana de Psicología, 16 (2), 125-137.

Daly, Louise; Sharek, D. DeVries, J. Griffiths, C. Sheerin, F. McBennett, P. Higgins, A. (2016). "The impact of four family support programmes for people with a disability in ireland". Journal of Intellectual Disabilities. 19(1). 34-50 
Pandey, S.; Agarwal, S. (2013).” transition to adulthood for youth with disability: issues for the disabled child and family". Journal Of Humanities And Social Science. 17 (3). 41-45.

Cardenal, F.; (2014). "discapacidad, sexualidad y familia". Recuperado de http://repositoriocdpd.net:8080/handle/ $123456789 / 52$

Carroscosa, L.; (2014). "El papel de la familia en la salud de las personas mayores. Articulación entre las relaciones familiares y la discapacidad en Europa". Revista Vasca de Sociología y Ciencia Política. Recuperado de https://dialnet.unirioja.es/servlet/articul o? codigo $=4935957$

Madrigal, A.; (2015). "El papel de la familia de la persona adulta con discapacidad en los procesos de inclusión laboral: Un reto para la educación especial en Costa Rica". Revista Electronica Educare, 19 (2), 197-211.

Silva, T.;(2015.). "La familia de la persona con discapacidad mental: una intervención desde trabajo social". Revista de Trabajo Social. 5 (1). 113129

Sandhu, P.; Ibrahim, J. Chinn. (Marzo, 2016). " 'I Wanted to Come Here Because of My Child': Stories of Migration Told by Turkish-Speaking Families Who Have a Son or Daughter with Intellectual Disabilities". Journal of Applied Research in Intellectual Disabilities. Recuperado de http://www.ncbi.nlm.nih.gov/pubmed/ 26939564

Rea, A.; Acle, G. Rueda, A. (2014). "Caracterización de los conocimientos de las madres sobre la discapacidad de sus hijos y su vínculo con la dinámica familiar". Acta Colombiana de Psicología, 17 (1), 91-103.

Ortega, P.; Salguero, A. Garrido, G. (2007). "discapacidad: paternidad y cambios familiares". Avances en Psicología Latinoamericana. 25 (1). 118-125.

Guevara, Y.; González, E. (2012).’Las Familias Ante la Discapacidad". Revista Electrónica de Psicología Iztacala. 15 (3). 1023-150.

Gonzales, A.; Rueda, C. Cagigal, V. Blas, E. (2013). "la calidad de vida de las familias de personas con discapacidad intelectual". REOP. 24 (1). 93-109.

Rodríguez, A.; Verdugo, A. Cruz, M. (2008). "calidad de vida familiar y apoyos para los progenitores de personas con discapacidad intelectual en proceso de envejecimiento". Revista Española sobre Discapacidad Intelectual. 39 (3). 19-34.

Córdoba, D.; Portilla, M. Arteaga, G. (2010). "dinámica familiar interna e higiene bucal en personas con discapacidad intelectual leve". Hacia la Promoción de la Salud. 15 (2). 8193.

Alemán de león. 2015. El impacto en la discapacidad en la familia. Trabajo de fin de grado para optar al título en trabajo social. San Cristóbal de la Laguna. Tenerife. Universidad de la Laguna. 56 p.

Majarres, D.; (2012). "Apoyo y fortalecimiento a familias para la crianza de niños con discapacidad". Horizontes Pedagógicos. 14(1).97-118. 
Bosley, H.; Kozub, M.(2011). "family travel experiences when one member has a developmental disability". Proceedings of the Northeastern Recreation Research Symposium. Recuperado de http://scholarworks.umass.edu/cgi/vie wcontent.cgi? article $=1016 \&$ context $=$ n err

Manjarrés, D.; León, E. Martínez, R. Gaitán, A. (2013). "Crianza y discapacidad: Una visión desde la vivencias y relatos de las familias en varios lugares de Colombia". Universidad Pedagógica Nacional. Colombia. 126 p.

Peralta, F.; Arellano, A. (2016). “El papel de la familia en la promoción de la autodeterminación de sus hijos/as con Síndrome de Down: el enfoque centrado en la familia (ECF)". No tiene revista, no tiene volumen, no tiene número. 1-14.

Lamigueiro, M.; (2014). "Impulsar la formación en autodeterminación de familiares de personas con discapacidad intelectual o del desarrollo". Revista Española Sobre Discapacidad Intelectual. 45 (250), 6884. Recuperado de https://dialnet.unirioja.es/servlet/articul $\mathrm{o}$ ? codigo $=5203319$

Romero, C. Peralta, S. 2012. Estudio de la dinámica en familias con hijos/as con síndrome de Down. Centro de investigación y difusión de la carrera de Psicología. Paraguay. Eureya 9 (1): $69-77 \mathrm{p}$.

Peralta, F.; Arellano, A. (2010). "familia y discapacidad una perspectiva teórico-aplicada del enfoque centrado en la familia para promover la autodeterminación”. Electronic Journal of research in Educational Psychology. 8 (3). 1339-1362.

Marrugat, M.; (2005). "La familia cuidadora y su incidencia en la calidad de vida de las personas dependientes". Revista Multidisciplinar Gerontología. 15 (3). 171-177.

Seliner, B.; Latal, B. Spirig, R. (2016). "Experiences and support needs of parents of hospitalized children with multiple disabilities: a qualitative study". Pflege, 29 (2). Recuperado de http://www.ncbi.nlm.nih.gov/pubmed/ 26974279

Verdugo, M. (2000). Calidad de Vida en las Familias con Hijos con Discapacidad Intelectual. Instituto Universitario de Integración en la Comunidad. Colección FEAPS. España. Universidad de Salamanca.

Neely, S.; Dia, D. (2004). "families of children with disabilities: a review of literature and recommendations for interventions". Journal of Early and Intensive Behavior Intervention. 5 (3). 93-107.

Fernández, E.; Arias, B. Gómez, L. Jarrín, I. (2012). "Calidad y Vida en Familias de Personas Mayores con Discapacidad Intelectual". Revista Española Sobre Discapacidad Intelectual 43 (4), 31-48.

Arellano, A.; Peralta, F. (2015). "El enfoque centrado en la familia, en el campo de la discapacidad intelectual ¿cómo perciben los padres su relación con los profesionales?". Revista de investigación educativa. 33 (1). 119132. 
World Health Organization; (2010). "Better health, better lives: children and young people with intellectual disabilities and their families". Recuperado de http://www.euro.who.int/_data/assets/ pdf_file/0008/126566/e94426.pdf

Giné, C.; Balcells, A. Pinatella, D. Roura, J. Hernández, M. Mas, J. Salines, M. (2011). "Necesidades de apoyo de las familias de personas con discapacidad intelectual de Catalunya". Revista Española Sobre Discapacidad Intelectual. 42 (240). 31-49. Recuperado de https://dialnet.unirioja.es/servlet/articul o? codigo $=3806990$

Brown, M.; Hoyle, L. Karatzias, T. (Febrero, 2016). "The experiences of family carers in the delivery of invasive clinical interventions for young people with complex intellectual disabilities: policy disconnect or policy opportunity?". 25 (3-4). Recuperado de http://www.ncbi.nlm.nih.gov/pubmed/ 26818378

Pava, N.; (2015). "Narrativas conversaciones con familias y docentes de niños y niñas con discapacidad: un aporte metodológico". Interdisciplinaria, 32 (2), 203-222.

Sáinz, F.; (2003) "Memoria y esperanza de las familias de personas con discapacidad" Revista Española Sobre Discapacidad Intelectual. 34 (207). 2938. Recuperado de https://dialnet.unirioja.es/servlet/articul $\mathrm{o}$ ?codigo $=738421$

Mas, J.; Giné, C. (2012). “Adaptación transcultural de la "Ecocultural Family Interview" a las familias con un hijo con discapacidad intelectual de Cataluña". Revista Española Sobre Discapacidad Intelectual. 43 (242). 26$45 . \quad$ Recuperado de https://dialnet.unirioja.es/servlet/articul o? codigo $=3911724$

Lagary, C.; Sanders, F. Brophy, L. (2015). "implications for family carers when people with psychosocial disability have individualised funding packages - literature review". University of Melbourne, Australia. 44.

García, J.; Cándido, J. Saura, I. Gonzálvez, C. Vicent, M. Gómez, I. (2013). "Familia y discapacidad intelectual, un estudio bibliométrico de 2000-2011 a través de la Social Science Citation Index". Revista Española Sobre Discapacidad Intelectual. 44 (246). 67-82. Recuperado de https://dialnet.unirioja.es/servlet/articul o? codigo $=4269828$

Araya, S.; (2007). "De lo invisible a lo cotidiano: familias y discapacidad". Actividades Investigativas en Educacion. 7 (3). 1-21. 


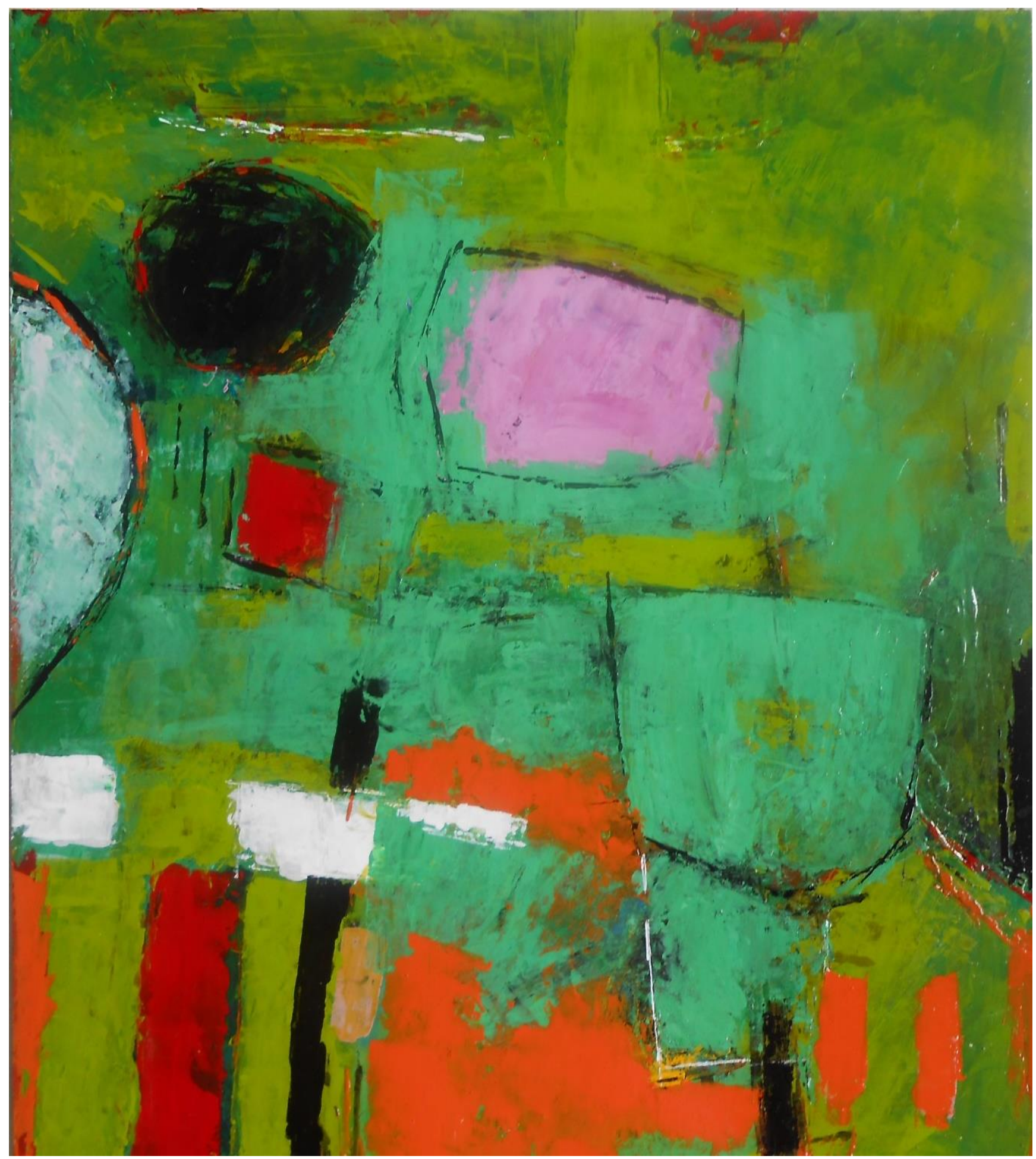

Título de la obra: Espacio vital-arqueología y paisaje

Técnica: Pigmentos industriales sobre polietileno

Dimensiones de la obra: $0,50 \mathrm{cms} \times 0,50 \mathrm{cms}$

Año: 2014

Nombre de la imagen adjunta: $O O 2$ 